Análisis y comentarios

Volumen 32(2):650-661. Mayo-agosto, 2021

e-ISSN 2215-3608, doi:10.15517/am.v32i2.42041

https://revistas.ucr.ac.cr/index.php/agromeso/index

\title{
El garbanzo en el occidente de Cuba: aportes de las ferias de agrodiversidad ${ }^{1}$
}

\section{Chickpea in western Cuba: Agrodiversity fairs contributions}

\author{
Regla María Cárdenas-Travieso², Rodobaldo Ortiz-Pérez², Dania Vargas-Blandino²
}

1 Recepción: 4 de junio, 2020. Aceptación: 22 de octubre, 2020. Este trabajo formó parte del Proyecto Innovación Agropecuaria Local (PIAL) financiado por la Agencia Suiza de Cooperación para el Desarrollo; Instituto Nacional de Ciencias Agrícolas, San José de las Lajas, Mayabeque, Cuba.

2 Instituto Nacional de Ciencias Agrícolas. San José de las Lajas, Mayabeque, Cuba. CP 32700. rmaria@inca.edu.cu (http://orcid.org/00000003-4667-4863), rortiz@inca.edu.cu (http://orcid.org/0000-0001-7266-7929), dania@inca.edu.cu (http://orcid.org/0000-0002-2084-4353).

\section{Resumen}

Introducción. El gobierno cubano, le ha concedido importancia a la producción nacional de garbanzo, con el propósito de sustituir importaciones, satisfacer las demandas del mercado y contribuir a la seguridad y soberanía alimentaria. Objetivo. Presentar los aportes de las ferias de agrodiversidad en la selección de nuevos genotipos de garbanzo (Cicer arietinum) en el occidente de Cuba. Materiales y métodos. El trabajo se desarrolló durante el periodo 2000-2018. Se analizó la selección de materiales de garbanzo de seis viveros introducidos por el Instituto Nacional de Ciencias Agrícolas (INCA) desde el Centro Internacional para Investigaciones Agrícolas en Zonas Áridas (ICARDA) y nueve variedades comerciales donadas por el Instituto de Investigaciones Fundamentales en Agricultura Tropical (INIFAT). Se sembraron en tres localidades de la región occidental de Cuba. Se realizaron ferias de diversidad para efectuar la selección de los genotipos con mejor comportamiento agroproductivo. Resultados. La comparación entre los viveros internacionales no mostró diferencias agroproductivas significativas entre ellos. Las variables de mayor peso en la caracterización agroproductiva fueron: días a maduración, días a floración, número de ramas, altura de las plantas y rendimiento. La mayor cantidad de genotipos seleccionados en las ferias de agrodiversidad correspondieron al vivero Élite Internacional para América Latina. El rendimiento promedio de los materiales seleccionados estuvo por encima de 1,00 t ha-1 . Conclusiones. La realización de ferias de agrodiversidad de garbanzo, ha aportado diecisiete nuevos materiales con idóneo comportamiento productivo, culinario y adaptabilidad a las condiciones edafoclimáticas de diferentes localidades del occidente de Cuba.

Palabras clave: genotipos, rendimiento, vivero, seguridad alimentaria.

\begin{abstract}
Introduction. The Cuban government has attached importance to the national production of chickpea, with the purpose of substituting imports, meeting market demands and contributing to food security and sovereignty. Objective. To present the contributions of the agro-diversity fairs in the selection of new chickpea (Cicer arietinum) genotypes in western Cuba. Materials and methods. The work was developed during the period 2000-2018. The selection of chickpea materials from six nurseries introduced by the National Institute of Agricultural Sciences (INCA) from the International Center for Agricultural Research in Dry Areas (ICARDA) and nine commercial
\end{abstract}


varieties donated by the Institute for Fundamental Research in Tropical Agriculture (INIFAT) were analyzed. They were planted in three locations in the western region of Cuba. Agro-diversity fairs were held to select the genotypes with the best agricultural production performance. Results. The comparison between the international nurseries did not show significant agro- productive differences between them. The variables with the greatest weight in the agroproductive characterization were: days to maturation, days to flowering, number of branches, plant height, and yield. The largest number of genotypes selected in the agro-diversity fairs corresponded to the Elite International nursery for Latin American. The average yield of the selected materials was above $1.00 \mathrm{t} \mathrm{ha}^{-1}$. Conclusions. The holding of chickpea agro-diversity fairs has provided 17 new materials with suitable productive and culinary behavior and adaptability to the edaphoclimatic conditions of different locations in western Cuba.

Keywords: genotypes, yield, nursery, food security.

\section{Introducción}

Entre los objetivos de las políticas implementadas en Cuba con la finalidad de perfeccionar el modelo económico, político y social se destacan: garantizar la seguridad alimentaria y lograr, a mediano plazo, un grado superior de soberanía alimentaria, mediante la sustitución de importaciones en todos aquellos renglones con potencialidades de desarrollo nacionales.

El Proyecto de Innovación Agropecuaria Local (PIAL), que pertenece al Instituto Nacional de Ciencias Agrícolas (INCA), en sus años de trabajo con productores y productoras del país, ha demostrado que para lograr la seguridad y soberanía alimentaria es necesario generar mayores niveles de participación, potenciar sus capacidades creativas y de experimentación, así como posibilitar espacios de articulación, intercambio y construcción colectiva en los escenarios productivos (Dacal, 2020; Ortiz et al., 2015). Entre estos escenarios se encuentran las ferias de agrodiversidad.

Las ferias de agrodiversidad son encuentros que se realizan en el campo con la participación de agricultores, fitomejoradores, decidores y otros. Tienen el propósito de contribuir, a través de la selección participativa de variedades, en la obtención de líneas segregantes y otros, al mantenimiento e incremento de la biodiversidad de especies y variedades de cultivos de interés para los agricultores (Ortiz et al., 2015) y para la seguridad alimentaria de sus comunidades.

El PIAL se ha propuesto lograr incrementos en la diversidad de garbanzo, ya que es una legumbre que tiene un alto valor nutricional y es muy demandada por la población cubana para el consumo.

El garbanzo (Cicer arietinum) tiene la cualidad de ser tolerante a la sequía, por lo que su cultivo tiene importancia como medida de adaptación al cambio climático. Como el resto de las legumbres, contribuyen a disminuir el efecto invernadero, debido a que mejora la absorción de carbono de la atmosfera a través de la fotosíntesis. En la rizósfera del garbanzo se establece una simbiosis natural con bacterias del género Rhizobium y Bradyrhizobium, que son capaces de atrapar el nitrógeno de la atmósfera en forma de óxido nitroso $\left(\mathrm{N}_{2} \mathrm{O}\right)$ y fijarlos en las raíces en forma de amoníaco $\left(\mathrm{NH}_{3}\right)$, sustancia que sí puede ser utilizada por el metabolismo de la planta durante los estados de crecimiento y desarrollo del grano. Esta propiedad les permite crecer con menos requerimientos de fertilizante nitrogenado, lo que contribuye a la reducción de emisiones de gases de efecto invernadero desde la agricultura.

En Cuba, desde el punto de vista agrícola, el garbanzo es un cultivo no tradicional y estacional, con un corto periodo óptimo de siembra (noviembre-diciembre), por lo que debe ajustarse a una buena planificación de la cosecha, con la finalidad de obtener altos niveles de producción (con bajos requerimiento de insumos) antes de que se inicie el periodo lluvioso, ya que tolera muy poco la humedad del suelo (Cárdenas et al., 2016). 
Aunque el garbanzo no es un cultivo tradicional en Cuba, se ha cultivado con frecuencia en la región oriental y central del país, donde las condiciones de aridez son más extremas, lo que ha resultado en una mayor cultura agraria en el cultivo de esta especie en estas regiones, con relación a la región occidental.

Las cualidades nutricionales y la tolerancia a la sequía del garbanzo, lo convierten en un cultivo muy prometedor para el occidente, donde se deben tomar acciones con la finalidad contribuir a la sustitución de importaciones y a la seguridad alimentaria y nutricional de la población.

El objetivo del presente trabajo es presentar los aportes de las ferias de agrodiversidad en la selección de nuevos genotipos de garbanzo (Cicer arietinum) en el occidente de Cuba.

\section{Consideraciones sobre la propuesta de incentivar el cultivo del garbanzo en Cuba}

En Cuba, desde el año 2008 se ha considerado la producción de alimentos como un asunto de seguridad nacional y se le ha concedido importancia al aumento de la producción de garbanzo, con el propósito de sustituir importaciones y satisfacer las demandas del mercado nacional.

El fundamento de las históricas importaciones de garbanzo se sustentaba en que es una legumbre muy apreciada por los cubanos para el consumo, tiene, además, un alto valor nutritivo. En consecuencia, en los últimos diez años Cuba ha importado garbanzo de varios países, pero en su mayoría provienen de México y Canadá, mientras que los Estados Unidos no han exportado garbanzos a Cuba desde 2011. Por lo tanto, su producción en el país constituye un valioso aporte al empeño de sustituir las importaciones de granos. Aunque los incrementos en las áreas de siembras son discretos y se concentran fundamentalmente en las provincias orientales, se aprecia una tendencia al aumento en otras zonas del país, lo que se sustenta en el conocimiento cada vez mayor del cultivo, el ajuste en los precios en la comercialización y sus bajos costos de producción con relación a otras especies de granos.

Para incentivar su cultivo, es recomendable que la gestión gubernamental favorezca la creación de circuitos locales de producción y consumo, que mejoren el acceso a este alimento a nivel local, para lo cual es importante reforzar y reconocer el papel académico y de la ciencia en la solución de lagunas de conocimiento y formulación de estrategias para alentar su cultivo y consumo, sin olvidar el conveniente abordaje en la difusión de productos comunicativos dirigidos a promover su inclusión en sistemas agrícolas sostenibles y dietas saludables.

\section{Antecedentes del cultivo de garbanzo en Cuba}

El garbanzo pertenece al grupo de las legumbres secas, cuyo grano es muy apreciado en la alimentación humana y animal por su valor nutritivo, posee entre un 18-25\% de proteínas. El modo de prepararlo está determinado por factores étnicos y regionales, que por su implicación sociocultural deben ser tenidos en cuenta en la planificación de la oferta/demanda en función del desarrollo local.

Para saber el por qué del cultivo y consumo de garbanzo en Cuba, es necesario conocer sus antecedentes, relacionados con su origen y cultivo en el mundo.

Estudios realizados a partir de 1975 consideraron que el centro de origen del garbanzo se ubica en la región del Creciente Fértil, más precisamente en el sureste de Turquía, donde podría haber comenzado el proceso de domesticación. Si bien, para algunos hallazgos se mencionaron diferencias en su antigüedad, es posible que sea debido al criterio de calibración utilizado para expresar la datación de los especímenes mediante la técnica de radiocarbono. Se considera al garbanzo como una de las especies pioneras o fundadoras de la agricultura, conjuntamente con la cebada (Hordeum vulgare), el trigo (Triticum monococcum subsp. monoccocum y T. turgidum subsp. dicoccum), la haba (Vicia faba), la lenteja (Lens culinaris), la arveja (Pisum sativum), la veza (Vicia ervilia) y el lino (Linum usitatissimum) (Biderbost, 2016). 
Sobre la presencia del garbanzo en América, se tienen referencias a partir del segundo viaje de Colón en 1493, como la primera especie introducida y cultivada por los españoles en el continente americano; fue sembrado en La Isabela (isla La Española, República Dominicana) y estaba fructificando en marzo de 1494.

En Cuba, se tienen noticias del cultivo del garbanzo en un censo realizado en 1827, donde se hizo referencia al cultivo de esta especie en el antiguo departamento del Centro (actualmente región central de la isla) (Sarmientos, 2001). No obstante, fuentes recientes sitúan en 1968 los primeros intentos de producción en Cuba por un campesino de procedencia canaria, en la localidad de mallorquín de la zona de Velasco, perteneciente al municipio de Gibara en la provincia de Holguín, quien comenzó a cultivar este grano en ese año (Quintero et al., 2013). A partir de los primeros años del siglo XXI, se encontraron las experiencias más documentadas de su cultivo en Cuba (Cárdenas et al., 2016; Meriño et al., 2017; Shagarodsky et al., 2001; Quintero et al., 2013). Las producciones se concentran de manera sostenida en los agricultores del Valle de Caujerí en Guantánamo, Las Tunas y Holguín en la región oriental de Cuba y en forma no sistemática en otras regiones como la central en Las Villas, en la zona de Matagua, Banao en Sancti Spíritus.

En la década del 80 en Güira de Melena, en la región occidental, se logró cultivar garbanzo con éxito en la Estación N.I. Vavilov, con semillas procedentes de material importado. Estas experiencias son resultado del quehacer científico de varias instituciones agropecuarias, de ahí que en los últimos cuarenta años se han evaluado una gran diversidad de genotipos mejorados para diferentes estreses. Solo se han registrado nueve variedades en la lista oficial de variedades comerciales: Nacional-6, Nacional-24, Nacional-27, Nacional-29, Nacional-30, Nacional 5HA, Blanco Sinaloa 92, Jamu 96 y JP-94 (Ministerio de la Agricultura, 2017). Esta baja diversidad limita la disponibilidad de cultivares que respondan a diversos requerimientos edafoclimáticos.

El consumo del garbanzo en Cuba es el resultado del proceso de transculturación culinaria influenciado por la cocina española. La aproximación a la génesis de la cocina cubana resalta la implantación entre 1900-1920, de la cocina galaico-asturiana en la gastronomía y la familia cubana con los famosos potajes con garbanzo, fabadas y caldos gallegos. Al parecer de esa época datan los cocidos de garbanzo que persisten hasta nuestros días, aunque probablemente se consumieran desde antes del siglo XIX, si se tiene en cuenta la referencia a su cultivo en el censo de 1827 (Sarmientos, 2001).

Si bien en Cuba el garbanzo se consume casi exclusivamente en forma de grano, resulta vital diversificar su consumo y promover la elaboración de productos derivados como: harina, pasta (humus) y confituras e ingredientes para diversas preparaciones gastronómicas. También se puede emplear el garbanzo en la alimentación animal, la cubierta del grano, las hojas y los tallos verdes y secos son usados para alimentar al ganado en muchos países en desarrollo, además toda la semilla puede ser molinada directamente para este propósito.

\section{El cambio climático: contribución del cultivo de garbanzo}

Los impactos del cambio climático (CC) son más significativos en países tropicales como Cuba, donde los riesgos se acentúan como consecuencia de su condición insular, su configuración alargada y estrecha, así como la posición longitudinal que ocupa en el Mar Caribe, siendo el clima cálido tropical estacionalmente húmedo, con influencia marítima.

Dentro de los sectores económicos, la agricultura es uno de los más afectados por el CC, debido entre otros factores, a la inmovilidad de las plantas de cultivo, a su estrecha interacción con el ambiente y a las consecuencias negativas del CC sobre el recurso suelo, conducente a la aridez. Con referencia a lo anterior, el estado cubano ha elaborado un Plan de Estado para el enfrentamiento al cambio climático denominado Tarea Vida, que entre sus acciones estratégicas recomienda adaptar las actividades agropecuarias, en particular las de mayor incidencia en la seguridad alimentaria del país, a los cambios en el uso de la tierra, al manejo de variedades adaptadas a las condiciones locales y al uso de insumos no contaminantes. 
Los agricultores deben prestar mucha atención a las formas más adecuadas de hacer frente a los posibles riesgos (sequías, salinidad, y altas temperaturas), a medida que los sistemas de producción de alimentos se transforman para adaptarse a las condiciones climáticas cambiantes. Esto implica realizar cambios en el paradigma agroproductivo; una buena idea es enfocar la atención en cultivos alternativos, como el garbanzo, que pueden ser incorporados a los necesarios sistemas de rotación con los cultivos tradicionales, que contribuyen a diversificar la producción y la economía con sistemas de reducido laboreo y riego, lo que conduce a una agricultura menos agresiva para los suelos y el ambiente.

\section{Aportes del Proyecto de Innovación Agropecuaria Local (PIAL) a la introducción y dise- minación de nuevos cultivares de garbanzo}

Con el inicio del fitomejoramiento participativo (FP) en Cuba en 1999, comenzaron a realizarse las ferias de agrodiversidad, que posteriormente, se sistematizaron (o institucionalizaron) con el apoyo del Proyecto de Innovación Agropecuaria Local (PIAL) (Ortiz et al., 2015), donde la selección participativa de variedades (SPV) ${ }^{3}$ ha sido una herramienta que ha contribuido al incremento de la diversidad vegetal en el sector agrícola a nivel local. La plataforma de trabajo del PIAL, se ha sustentado en dos variantes: una encaminada a la diversificación varietal de especies de importancia alimentaria, como los granos básicos: arroz, frijol y maíz, y otra dirigida a la introducción de especies mejoradas para diferentes estreses, como es el caso del garbanzo.

La implementación de nuevas formas de aprendizaje en la acción con la creación de nuevas capacidades interinstitucionales y humanas, para manejar procesos innovativos (Romero \& Ortiz, 2017) es esencial para estimular la selección, mantenimiento y diseminación de diversidad vegetal. Ejemplo de esto, son los dos ciclos de aprendizaje en la acción promovidos por el PIAL entre 2014 y 2019, relacionados con la producción local de semillas de calidad en varios municipios del país. Aunque los esfuerzos de estos ciclos están dirigidos a la capacitación para fortalecer la producción y comercialización local de semillas de granos básicos, colateralmente han favorecido el incremento en la producción local de semillas de otras especies de granos demandados por agricultores y consumidores, como el garbanzo y la soya (Glycine max.), cuya inclusión en los sistemas agrícolas del país puede contribuir a hacerlos más diversos, armónicos e integrales.

El PIAL con la intención de dejar capacidad instalada en los territorios, ha organizado también acciones formativas de recursos humanos, orientadas a escenarios locales, como el Diplomado en Sistema de Innovación Agropecuaria Local (SIAL), que incluye en su contenido el diseño de las ferias de agrodiversidad, para de esta forma, contribuir a su replicabilidad y sostenibilidad.

En garbanzo, las ferias de agrodiversidad han permitido la selección de genotipos promisorios a partir del conocimiento, gustos y necesidades de los productores y consumidores locales. Esto ha contribuido a la diversificación de la base genética de esta especie, con la adopción de nuevos cultivares adaptados a las condiciones locales y con mayor aceptación culinaria y comercial.

En los últimos diez años, ha sido evidente el impulso del PIAL a la ampliación de la base genética de esta especie con la importación de nuevos materiales procedentes del banco de germoplasma del International Center for Agricultural Research in the Dry Areas (ICARDA), en la República Árabe de Siria, que ha suministrado las colecciones internacionales de garbanzo mejorados para diferentes estreses, que han sido introducidas y evaluadas en diferentes localidades. Este accionar ha incentivado las investigaciones, la realización de ferias de agrodiversidad y festivales culinarios de garbanzo en la región occidental de la isla.

Participación de los productoresen la selección de variedades adaptadas a las características agroecológicas de sus fincas. 


\section{Codificación de las accesiones}

Los Bancos de Germoplasma suelen registrar las diferentes accesiones con códigos, en muchos casos difíciles de entender y retener por personas que tienen un mínimo de conocimientos en experimentación, como es el caso de los agricultores involucrados en el FP/PIAL. Para facilitar la adopción, retención y monitorear los materiales adquiridos, tanto por donaciones nacionales como internacionales, el PIAL adoptó una codificación más asequible a la diversidad de actores que interactúan con esos materiales (Cárdenas \& Ortiz, 2013). La codificación PIAL consta de tres elementos: especie-tipo de donación-número de la accesión en orden consecutivo:

En el caso del garbanzo los donativos se registran de dos formas según la procedencia de la donación: Ga-DNNo. o Ga-DI- No.

Donde:

Ga: garbanzo.

DN: donación procedente de instituciones nacionales.

DI: donación procedente de instituciones internacionales

No.: número de la accesión en orden consecutivo

La codificación utilizada es una buena práctica del PIAL que evidencia cambios de paradigmas en la búsqueda de una gestión más participativa de la experimentación agrícola. Se conserva la codificación original para la correspondiente devolución de información a los respectivos bancos emisores.

El nivel de adopción de las nuevas colecciones de garbanzo ha sido mayor en las provincias occidentales vinculadas al PIAL, lo que se explica por la articulación lograda entre instituciones y agricultores, intencionada con la realización de ferias de agrodiversidad que han incentivado la experimentación campesina en el cultivo de esta especie poco conocida en estas provincias. Además, de las facilidades que aporta la cercanía del centro emisor (INCA) a esos escenarios locales.

\section{Región occidental, sus características y resultados en el cultivo del garbanzo}

La Región occidental de Cuba, se encuentra ubicada entre los $22.4167^{\circ}, 23.0411^{\circ} \mathrm{N}$ y los $83.6967^{\circ}, 81.5775^{\circ} \mathrm{O}$. En esta región las temperaturas máximas medias varían entre 26 y $30^{\circ} \mathrm{C}$ y las temperaturas mínimas medias entre 18 y $22^{\circ} \mathrm{C}$. La temporada invernal es azotada por frentes fríos procedentes de América del Norte, que provocan una disminución de las temperaturas. En el periodo lluvioso (mayo-octubre) las precipitaciones fluctúan entre 1200 y 1400 $\mathrm{mm}$ y durante el periodo seco (noviembre-abril) entre los 200 y $400 \mathrm{~mm}$. En esta región se ubican cinco provincias, entre las que se encuentran Mayabeque, Artemisa y Pinar del Rio (municipio Los Palacios), analizadas en este trabajo.

En una investigación realizada por Cárdenas-Travieso et al. (2012) en San José de las Lajas, Mayabeque, para caracterizar morfo agronómicamente la colección importada, se mostró la no existencia de diferencias agroproductivas significativas entre los genotipos de cuatro viveros estudiados y las variables de mayor peso en la caracterización fueron: días a maduración, días a floración, número de ramas, altura y rendimiento.

Con la aprobación del INCA, el proyecto ha introducido desde el ICARDA, más de 380 accesiones de garbanzo de seis viveros: Resistencia a la Rabia (Ascochyta rabiei), Resistencia a la Fusariosis (Fusarium spp.), Resistencia al Minador de la hoja (Liriomyza spp.), Tolerancia a la sequía, Élite Internacional para América Latina y Latitudes Sureñas ${ }^{4}$.

En el vivero Elite Internacional para América Latina, siete genotipos promisorios promediaron un rendimiento superior a $400 \mathrm{~g} \mathrm{~m}^{-2}$. Se entregaron semillas de estos genotipos a diferentes instituciones y agricultores vinculados al PIAL, para la evaluación y selección participativa en las ferias de agrodiversidad de esta especie en sus fincas.

4 Importado con el objetivo de ampliar la base genética de la especie. 
La primera feria de agrodiversidad de garbanzo, organizada por los especialistas del PIAL, se realizó en el municipio San Antonio de los Baños de la provincia Artemisa en el año 2009. Se evaluaron veintiún genotipos procedentes de ICARDA y cinco variedades nacionales (Ministerio de la Agricultura, 2017), todos del tipo Kabul, donadas por el Instituto de Investigaciones Fundamentales en Agricultura Tropical “Alejandro de Humboldt" (INIFAT).

La selección participativa reveló que el porcentaje de diversidad efectiva fue 76,9 \%, lo que indicó que la mayoría de los materiales se adaptaron a las condiciones edafoclimáticas de la localidad (Cárdenas et al., 2016).

Los cultivares no seleccionados fueron afectados por el gusano de la cápsula (Heliothis virescens), considerado como la principal plaga insectil que afecta al cultivo en América, y en Cuba se ha informado sus daños en plantaciones del oriente cubano (Pérez \& Suris, 2011).

Los criterios más utilizados (Cuadro 1) para seleccionar los cultivares de interés fueron: vigor vegetativo (con categoría de bueno a muy bueno en el $80 \%$ de los cultivares seleccionados), número de vainas por planta (70 \% de los cultivares seleccionados presentaron más de 115 vainas por planta) y tamaño del grano (50\% de los cultivares elegidos presentaron granos grandes con masa superior a $40 \mathrm{~g} / 100$ granos). Estos criterios alcanzaron valores por encima del promedio de votos emitidos (Cárdenas et al., 2016).

Cuadro 1. Criterios utilizados por los participantes durante la evaluación participativa de veintiséis líneas de garbanzo (Cicer arietinum). Cooperativa de Producción Agropecuaria (CPA) “Gilberto León”, San Antonio de Los Baños, Artemisa, Cuba. 2010.

Table 1. Criteria used by participants during the participatory evaluation of 26 chickpea (Cicer arietinum) lines. Agricultural Production Cooperative (CPA) “Gilberto León”, San Antonio de Los Baños, Artemisa. Cuba 2010.

\begin{tabular}{lc}
\hline Criterios & Cantidad de votos \\
\hline Total de votos & 175 \\
• Vigor vegetativo & 42 \\
- Vainas por planta & 34 \\
- Tamaño grano & 22 \\
- Porte & 17 \\
- Altura & 14 \\
- Sanidad & 14 \\
- Granos por vaina & 11 \\
- Población & 11 \\
- Ciclo & 9 \\
- Maduración & 1 \\
Promedio de votos & 17,5 \\
\hline
\end{tabular}

Se concedió un mayor número de votos a los cultivares Ga-DI-61, Ga-DI-18 y Ga-DI-21, con diferencias significativas entre ellos, el primero, con la máxima votación, pertenece al vivero de resistencia al Tizón por Ascochyta (A. rabiei), mientras los otros dos son del vivero Elite Internacional para América Latina.

En la provincia Mayabeque, se evaluaron once genotipos foráneos avanzados de garbanzo en fincas del municipio San José de las Lajas (campaña de frío ${ }^{5}$ 2016/2017). Entre ellos, nueve líneas del tipo Kabuli de testa color crema, conservadas en el banco de germoplasma del INCA. También se evaluaron dos cultivares tipo Desi de testa oscura: Negro IEC de testa negra y WR-315 de color pardo, donados por el INIFAT de Cuba.

5 Comprendida tradicionalmente entre los meses de noviembre a febrero. 
En la feria de agrodiversidad realizada en San José de las Lajas, se seleccionaron dos genotipos (Ga-DI-30 y Ga-DI-34) del vivero Élite Internacional para América Latina, los cuales se sembraron en la campaña 2017/2018, junto a Ga-DI-186 y la variedad comercial Blanco Sinaloa (Cuadro 2), en pequeñas áreas de la finca El Mulato de la Cooperativa de Créditos y Servicios (CCS) Orlando Cuellar, las cuales pasaron a formar parte de la colección conservada por el agricultor, en el banco local de semillas situado en dicha finca.

Cuadro 2. Producción y rendimiento de genotipos de garbanzo (Cicer arietinum) cosechados en Mayabeque, campaña $2017 / 2018$. Cuba.

Table 2. Production and yield of chickpea (Cicer arietinum) genotypes harvested in Mayabeque, campaign 2017/2018. Cuba.

\begin{tabular}{lcccc}
\hline & \multicolumn{3}{c}{ Genotipos } \\
\cline { 2 - 5 } Indicadores & Ga-DI-30 & Ga-DI-34 & Ga-DI-186 & Blanco Sinaloa \\
\hline Área cosechada (ha) & 0,20 & 0,15 & 0,03 & 0,02 \\
Producción (kg) & 200,00 & 193,18 & 31,82 & 6,82 \\
Rendimiento $\left(\mathrm{kg} \mathrm{ha}^{-1}\right)$ & 1020,41 & 1277,66 & 946,97 & 347,87 \\
\hline
\end{tabular}

El rendimiento obtenido con tecnología de bajos insumos en los genotipos Ga-DI-30 y Ga-DI-34, superó 1,0 t ha-1 y se puede considerar aceptable, si se tiene en cuenta que en Argentina, país productor y exportador de esta leguminosa, se han registrado promedios de rendimiento de $1,8 \mathrm{t} \mathrm{ha}^{-1}$ con un rango entre 1,2 y 2,5 $\mathrm{t} \mathrm{ha}^{-1}$ (Agrositio, 2012).

En el municipio Los Palacios, de la provincia Pinar del Rio, se ha evaluado y seleccionado, de acuerdo a su comportamiento agronómico, cinco nuevos genotipos importados (Ga-DI-1 a Ga-DI-5). Adicionalmente, se han realizado estudios fitosanitarios para la identificación de especies de Fusarium spp., agente causal de la Marchitez o Fusariosis, que es la enfermedad más importante del cultivo, la cual incide de forma negativa sobre los componentes del rendimiento y la calidad de la semilla.

El empleo de dos cepas del hongo antagonista Trichoderma asperellum, como alternativa biológica para el control de la Fusariosis, ha demostrado ser significativamente efectiva en la disminución de la enfermedad, el tratamiento al suelo, la semilla y el follaje, mostró resultados alentadores. El rendimiento y sus componentes, fueron significativamente mayores con este tratamiento, lo que repercutió en un mayor aporte en la relación beneficio-costo (Echevarría, 2014).

Las acciones emprendidas por el PIAL, en esta especie, sirven de base para la adopción, adecuación e implementación del cultivo sostenible del garbanzo en Cuba.

\section{Festivales culinarios, una herramienta para evaluar la calidad culinaria de genotipos promisorios de garbanzo}

Las pruebas de calidad de consumo, en genotipos promisorios, se realizan en la postcosecha, cuando se obtiene suficiente grano para estas determinaciones, en este caso, los agricultores y consumidores cuentan con disímiles criterios para evaluar y seleccionar, mediante encuestas, las variedades de su agrado, entre los criterios más utilizados están el tiempo de cocción, el sabor y cuajado del caldo.

Los festivales culinarios se convierten en marco propicio para estas pruebas degustativas, donde la participación de miembros de la comunidad, de género y edad diversa, garantizan la adopción de variedades mejoradas con mayor eficiencia y responsabilidad compartida. 
De manera general, las degustaciones realizadas a los granos de once genotipos importados desde ICARDA (GaDI-8, 27, 30, 34, 74, 133, 186, 191 y 242) y dos donados por INIFAT (Negro IEC y WR-315), han mostrado aceptables atributos culinarios para la elaboración del caldo. La valoración (utilizando los puntajes alto, medio, bajo) ha calificado la integridad del grano y la firmeza de la piel con una alta correlación positiva entre ambos, mientras que la presencia de pieles sueltas, dureza de la piel, mantecosidad, granulosidad, harinosidad, han calificado como media, en tanto, el sabor extraño ha sido valorado como bajo, este último altamente correlacionado con la dureza del grano (Cuadro 3).

Cuadro 3. Matriz de correlaciones $(\mathrm{p} \leq 0,05)$ de la valoración culinaria del garbanzo (Cicer arietinum) en festival culinario de San José de las Lajas, Cuba. 2016.

Table 3. Correlation matrix ( $\mathrm{p} \leq 0,05$ ) of the culinary valuation of chickpea (Cicer arietinum) at the San José de las Lajas culinary festival, Cuba. 2016.

\begin{tabular}{lcccccccc}
\hline & $\begin{array}{c}\text { Tiempo de } \\
\text { cocción }\end{array}$ & $\begin{array}{c}\text { Integridad } \\
\text { grano }\end{array}$ & $\begin{array}{c}\text { Pieles } \\
\text { sueltas }\end{array}$ & $\begin{array}{c}\text { Firmeza } \\
\text { piel }\end{array}$ & Dureza & Mantecosidad & $\begin{array}{c}\text { Granulosi- } \\
\text { dad }\end{array}$ & $\begin{array}{c}\text { Harinosi- } \\
\text { dad }\end{array}$ \\
\hline Integridad & & & & & & & & \\
grano & $-0,5033$ & & & & & & & \\
pieles sueltas & 0,5594 & $-0,2436$ & & & & & & \\
firmeza piel & $-0,2875$ & $\mathbf{0 , 8 5 6 0}$ & $-0,0131$ & & & & & \\
dureza & $-0,4624$ & $-0,1556$ & $-0,2430$ & 0,0191 & & & & \\
mantecosidad & 0,0344 & 0,2802 & 0,2976 & 0,6940 & 0,4172 & & & \\
granulosidad & $-0,0506$ & 0,2686 & $-0,1444$ & 0,2216 & 0,3938 & 0,2437 & & \\
harinosidad & $-0,0506$ & 0,2686 & 0,4604 & 0,2216 & 0,1926 & 0,2437 & 0,6865 & \\
sabor extraño & $-0,4841$ & $-0,0139$ & $-0,5781$ & 0,0946 & $\mathbf{0 , 8 9 1 1}$ & 0,2784 & 0,4783 & 0,0031 \\
\hline
\end{tabular}

Las encuestas han permitido constatar, además, la tradición popular en la elaboración del garbanzo, de remojar el grano durante al menos ocho horas (generalmente durante la noche), para cocinarlo al siguiente día, en olla a presión ${ }^{6}$ (recipiente hermético para cocinar que puede alcanzar presiones más altas que la atmosférica, para reducir el tiempo de cocción). Como resultado de las ferias de agrodiversidad, festivales culinarios e investigaciones realizadas, se han podido seleccionar diecisiete nuevos genotipos promisorios para el occidente de Cuba (Cuadro 4).

Desde el ámbito generacional, el análisis de la composición por edades ha mostrado una baja participación de jóvenes en la elaboración doméstica de esta legumbre. Un ejemplo de esto se puede apreciar en la Figura 1, donde los premiados en el festival culinario de San José de las Lajas comprenden las edades desde 45 a 74 años. Esta situación puede contrarrestarse con la realización sistemática de ferias de agrodiversidad y festivales culinarios de garbanzo en diferentes localidades, diseñados como actividades socioculturales para motivar y comprometer a los jóvenes con la elaboración de esta legumbre.

En España, según una nota del diario independiente de Asturias La nueva España, ya existe preocupación porque cada vez menos jóvenes saben hacer el potaje de garbanzo, aún cuando este es uno de los platos más tradicionales para la Semana Santa.

6 En Cuba el uso masivo de olla a presión para la cocción de alimentos es una de las acciones llevadas a cabo por el gobierno para promover el uso racional de la energía y la protección del ambiente. Se traduce, en una mejoría en la calidad de vida, por cuanto la cocción resulta más saludable y contribuye a mitigar los impactos ambientales debido a la reducción de emisiones de gases de efecto invernadero, resultando ventajoso con respecto a la cocción lenta con biomasa (leña, carbón y otros) común en escenarios rurales. 
Cuadro 4. Nomenclatura de los genotipos de garbanzo (Cicer arietinum), importados del International Center for Agricultural Research in the Dry Areas (ICARDA) evaluados y seleccionados por el Instituto Nacional de Ciencias Agrícolas (INCA)/ Proyecto de Innovación Agropecuaria Local (PIAL), durante los años del 2007 al 2013. Cuba.

Table 4. Nomenclature of chickpea (Cicer arietinum) genotypes imported from the International Center for Agricultural Research in the Dry Areas (ICARDA), evaluated and selected for the Instituto Nacional de Ciencias Agrícolas (INCA)/Local Agricultural Innovation Project (PIAL) during the years 2007 to 2013. Cuba.

\begin{tabular}{cccc}
\hline Código PIAL & Nombre ICARDA & Código PIAL & Nombre ICARDA \\
\hline Ga-DI-1 & FLIP 00-70C & Ga-DI-30 & FLIP 04-15C \\
Ga-DI-2 & FLIP 01-26C & Ga-DI-34 & FLIP 88-85C \\
Ga-DI-3 & FLIP 02-03C & Ga-DI-61 & FLIP 03-73C \\
Ga-DI-4 & FLIP 03-22C & Ga-DI-74 & FLIP 04-25C \\
Ga-DI-5 & FLIP 97-102C & Ga-DI-133 & LMR 134 \\
Ga-DI-8 & FLIP 01-29C & Ga-DI-186 & FLIP06-30C \\
Ga-DI-18 & FLIP 03-110C & Ga-DI-191 & FLIP06-67C \\
Ga-DI-21 & FLIP 03-120C & & FLIP06-127C \\
Ga-DI-27 & FLIP 04-1C & \\
\hline
\end{tabular}

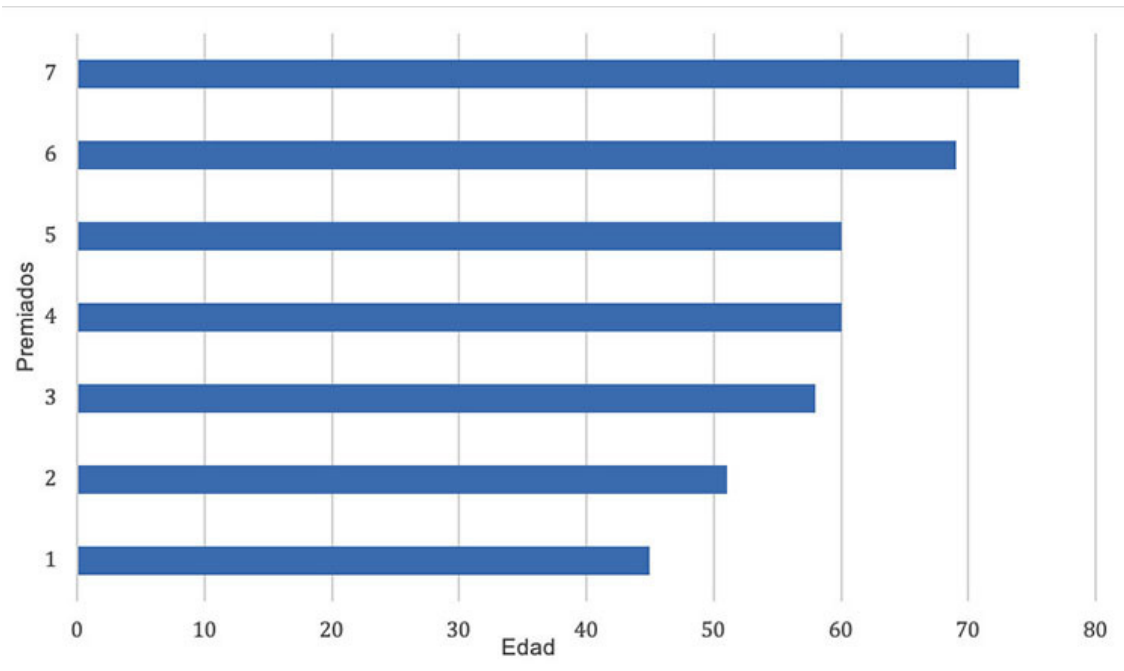

Figura 1. Edades de los participantes premiados en festival culinario de San José de las Lajas, Mayabeque, Cuba. 2017.

Figure 1. Ages of the awarded participants in the culinary festival of San José de las Lajas, Mayabeque, Cuba. 2017.

\section{Conclusiones y recomendaciones}

La realización de ferias de agrodiversidad, ha aportado diecisiete nuevos materiales con adecuado comportamiento productivo, culinario y adaptabilidad a las condiciones edafoclimáticas de diferentes localidades del occidente de Cuba. 
Se recomienda realizar acciones encaminadas a: a) dar continuidad a los ciclos de aprendizajes en la acción que favorezcan la capacitación en producción y comercialización local de semillas de garbanzo; b) continuar con la realización de ferias de agrodiversidad y festivales culinarios, que permitan realizar la selección participativa de nuevas variedades con atributos productivos, culinarios y comerciales apropiados para cada localidad; y c) la elaboración de productos comunicativos que aborden los beneficios del cultivo y consumo de garbanzo.

\section{Referencias}

Agrositio (2012, noviembre 07). Garbanzo: un cultivo rentable con pérdidas del $9 \%$ por cosecha ineficiente. http://www. agrositio.com.ar/noticia/138511-garbanzo-un-cultivo-rentable-con-perdidas-del-9-por-cosecha-ineficiente

Biderbost, E. (2016). Domesticación del garbanzo (Cicer arietinum L). Su introducción en Argentina. En J. Carreras, V. Mazzuferi, \& M. Karlin, (Eds.), El cultivo de garbanzo en Argentina (pp.19-38). Universidad Nacional de Córdoba. https://rdu.unc.edu.ar/handle/11086/6010.

Cárdenas, R. M., \& Ortiz, R. (2013). Tipificación y descripción de la diversidad manejada en instituciones vinculadas al Programa de Innovación Agropecuaria Local. En R. Ortiz (Ed.), La Biodiversidad Agrícola en manos del campesinado cubano (pp. 28-37). Instituto Nacional de Ciencias Agrícolas.

Cárdenas, R. M., de La Fé, C. F., Echevarría, A., Ortiz, R., \& Lamz, A. (2016). Selección participativa de cultivares de garbanzo (Cicer arietinum L.) en feria de diversidad de San Antonio de los Baños, Artemisa, Cuba. Cultivos Tropicales, 37 , 134-140. https://doi.org/10.13140/RG.2.1.1419.4161

Cárdenas-Travieso, R. M., Ortiz-Pérez, R., Echevarría-Hernández, A., \& Shagarodsky-Scull, T. (2012). Caracterización y selección agroproductiva de líneas de garbanzo (Cicer arietinum L.) introducidas en Cuba. Cultivos Tropicales, 33, $69-74$.

Dacal, A. (2020, octubre 12). Soberanía alimentaria: una urgencia pendiente (II). https://oncubanews.com/cuba/soberaniaalimentaria-una-urgencia-pendiente-ii/

Echevarría, A. (2014). Identificación y alternativa biológica para el control de la Marchitez en el cultivo del garbanzo (Cicer arietinum L.) [Tesis de Maestría no publicada], Universidad Agraria de La Habana.

Ministerio de la Agricultura. (2017). Lista oficial de variedades comerciales 2017-2018. https://www.minag.gob.cu/sites/ default/files/publicaciones/lista_oficial_de_variedades_comerciales_2017-2018.pdf

Meriño, Y., Boicet, T., Boudet, A., \& Cedeño, A. (2017). Respuesta agronómica de dos cultivares de garbanzo (Cicer arietinum L.) bajo diferentes condiciones de humedad del suelo en la provincia de Granma. Centro Agrícola, 44(2), 22-28. http://cagricola.uclv.edu.cu/index.php/es/volumen-44-2017/numero-2-2017

Ortiz, R., Miranda, S., Rodríguez, O., Gil, V. D., Márquez, M., \& Guevara, F. (2015). Las ferias de agrodiversidad en el contexto del fitomejoramiento participativo-programa de innovación agropecuaria local en Cuba. Significado y repercusión. Cultivos Tropicales, 36, 124-132.

Pérez, J., \& Suris, M. (2011). Insectos asociados al cultivo del garbanzo (Cicer arietinum L.) en la provincia Las Tunas. Protección Vegetal, 26, 191-193.

Quintero, E., Gil, V.D., Álvarez U., García, J.C., Andreu, C., \& Díaz, M. (2013). Potencialidades del garbanzo (Cicer arietinum L.) en Villa Clara en la producción diversificada de granos. Revista Centro Agrícola, 40(4), 73-78. http://cagricola. uclv.edu.cu/index.php/es/volumen-40-2013/numero-4-2013

660 
Romero, M. I., \& Ortiz, R. (2017). Hacia una gestión participativa del Desarrollo Local. Textos de apoyo al Diplomado para la implementación del Sistema de Innovación Agropecuaria Local. Ediciones Instituto Nacional de Ciencias Agrícolas.

Sarmientos, I. (2001). La alimentación cubana (1800-1868): producción interna e importaciones. Anales del museo de América, 9, 107-128.

Shagarodsky, T., Chiang, M. L. \& López, Y. (2001). Evaluación de cultivares de garbanzo (Cicer arietinum L.) en Cuba. Agronomía Mesoamericana, 12(1), 95-98. https://doi.org/10.15517/am.v12i1.17298 